\title{
Pallidal Signal Intensities on T1-weighted MRI are Highly Observed in Advanced Liver Cirrhosis
}

\author{
Young Cheol Weon, Neung Hwa Park*, Yangho Kim**广, and Heun Lee** \\ Department of Radiology, Ulsan University Hospital, University of Ulsan College of Medicine, Ulsan, Korea \\ * Department of Internal Medicine, Ulsan University Hospital, University of Ulsan College of Medicine, Ulsan, Korea \\ **Department of Occupational and Environmental Medicine, Ulsan University Hospital, \\ University of Ulsan College of Medicine, Ulsan, Korea
}

\begin{abstract}
Background: Manganese (Mn) has been found to increase the signal intensity of the globus pallidus (GP) on T1-weighted magnetic resonance images (MRI). We performed this study in order to determine the features of liver disease that correlate with pallidal signal intensities.

Methods: We assessed blood Mn levels and pallidal signals in T1-weighted MRI in 49 patients with liver cirrhosis and 23 healthy controls.

Results: Increased signal intensity in the GP was observed in 30 of 49 (61.2\%) patients with liver cirrhosis, with the pallidal index (PI) in patients with Child-Pugh classes B and C differing significantly from the PI in controls. Multiple linear regression analysis showed that blood Mn concentrations and Child-Pugh scores in cirrhotics were significantly associated with increased PI after controlling for other confounders $(p<0.05$ each).

Conclusions: Pallidal signals on T1-weighted MRI are mainly observed in advanced liver cirrhosis. The present study suggests that advanced liver cirrhosis may be a human model for manganism.
\end{abstract}

Keywords: manganese, signal intensity, magnetic resonance, liver cirrhosis, pallidal index

\section{Introduction}

Manganese ion $\left(\mathrm{Mn}^{2+}\right)$ has five unpaired electrons in the 3rd orbital, causing its large magnetic moment, which ultimately results in an increase in signal intensity on T1-weighted MRI, in particular, bilateral and symmetrical increases in signal intensity that are mainly confined to the globus pallidus (GP) and midbrain. ${ }^{1,2)}$ However, $\mathrm{Mn}^{2+}$ does not affect T2-weighted MRI, which is most sensitive for detecting brain pathology such as brain tumor or bleeding. These phenomena can be specifically used for pinpointing Mn accumulation in brain tissues. High signal intensity in the GP has been observed during experimental Mn poisoning of non-human primates, ${ }^{3)}$ in a patient with Mn intoxication, ${ }^{4)}$ and in workers exposed to $\mathrm{Mn}^{2)}$ Chronic liver failure has been associated with increased pallidal signal intensities on T1-weighted MRI, ${ }^{5-7)}$ with the correlation between MRI abnormalities in the GP and circulating $\mathrm{Mn}$ concentrations in patients with liver cirrhosis suggesting that these high signals are due to paramagnetic $\mathrm{Mn}^{8}{ }^{89}$ Several studies have examined the relationships among MR abnormalities, the severity of liver disease, hyperbilirubinemia and the degree of portal systemic shunting, but the results were inconsistent. ${ }^{10-15)}$ Previous studies also showed wide variations in the prevalence of high pallidal signals (52-100\%). ${ }^{9,10-16)}$ It is not clear which features of chronic liver disease may affect pallidal signal intensities. We therefore examined pallidal signals in patients with liver cirrhosis and healthy

Corresponding author: Dept. of Occupational and Environmental Medicine, Ulsan University Hospital, Ulsan 682-060, Korea, Tel: +82-52-250-7281 Fax: +82-52-250-7289, E-mail: yanghokm@nuri.net Received: 19 June 2012, Revised: 12 July 2012, Accepted: 10 September 2012 
controls to determine the features of liver disease that correlate with pallidal signal intensities.

\section{Subjects and Methods}

\section{Subjects}

Male patients with clinically confirmed hepatitis B-induced cirrhosis were selected from hospital inpatients and from patients attending an ambulatory hepatology care clinic. A diagnosis of hepatitis Binduced liver cirrhosis was based on the results of patient questionnaires, combined with clinical, laboratory, endoscopic and ultrasonographic findings. Of the 55 patients who provided informed consent and were recruited, 6 were excluded because they had received transfusions due to previous GI bleeding. Transfusion may confound iron deficiencyinduced susceptibility to $\mathrm{Mn}$. The control group consisted of 23 normal healthy men who visited a health promotion center. These subjects had no history of liver disease. Both patients and controls had no occupational exposure to Mn. After receiving a detailed explanation of the study design and potential risks, all participants provided written informed consent. The procedures used in this study were approved by the institutional review board of Ulsan University Hospital.

\section{Questionnaires, laboratory testing, and analysis of Mn concentration}

Samples of heparinized venous blood and urine were collected. Each subject filled out a questionnaire on basic demographic information, smoking history, alcohol consumption, medications, and a detailed medical history. Laboratory tests aimed at detecting liver dysfunction and anemia included liver function tests, blood cell counts, hemoglobin, hematocrit. The severity of liver disease was assessed using the Child-Pugh scoring system, ${ }^{16}$ which includes as variables the presence or absence of ascites and encephalopathy, serum albumin and bilirubin concentrations, and prothrombin time. Each variable is scored from 1 to 3 points for increasing abnormality, with total scores of 5-6, 79, and 10-15 defined as Child-Pugh classes A, B, and $\mathrm{C}$, respectively, with $\mathrm{C}$ being the most severe.

Blood Mn concentrations were determined by flameless graphite furnace atomic absorption spectrophotometry (AAS; Spectra AA880-GTA 100,
Varian, Australia) using a standard addition method. ${ }^{17,18)}$ Aliquots of $0.1 \mathrm{ml}$ blood were diluted 20 -fold with $0.1 \% \mathrm{v} / \mathrm{v}$ Triton X-100, and $15 \mu \mathrm{l}$ samples were injected into the graphite furnace.

\section{Brain MRI}

MRI examinations were performed using a 1.5 Tesla system (Signa Horizon LX; GE Medical Systems; Milwaukee, WI, USA). The spin echo (SE) technique was applied with two acquisitions in the $\mathrm{T} 1$ weighted sequence and one acquisition in the $\mathrm{T} 2$ weighted sequence. $\mathrm{T} 1$ weighted images were acquired in the axial plane using the following parameters: 600/20 (repetition time $\left(T_{R}\right)$ / echo time $\left(T_{E}\right) \mathrm{m} \mathrm{sec}$ two excitations, a $22 \mathrm{~cm}$ field-of-view, a $256 \times 160$ matrix, and slice thickness of $5 \mathrm{~mm}$. T1 weighted sagittal images were obtained with the same parameters, except that $T_{R} / T_{E}$ was $466 / 14$ ms. Axial T2-weighted $\left(T_{R} /\right.$ $\mathrm{T}_{\mathrm{E}}=4000 / 100 \mathrm{~ms}$ ) images were also obtained. The signal intensity of the GP relative to that from frontal white matter was subjectively evaluated by a radiologist with no prior knowledge of patient status. High or increased signal intensity of GP was defined when pallidal signal intensity is higher than the intensity of frontal white matter. For quantitative evaluation of signal intensities, a region of interest (ROI) was placed in the region of highest intensities in the GP, as determined by visual assessment, and in the subcortical frontal white matter. Pallidal index (PI) was calculated as the ratio of signal intensity in the GP to that in the subcortical frontal white matter on axial T1weighted MRI, multiplied by $100{ }^{8)}$

\section{Statistical analyses}

Univariate distributions of continuous variables were examined to evaluate normality using the Kolmogorov-Smirnov test. To better attain normal distributions, blood Mn, serum AST (aspartate aminotransferase), and ALT (alanine aminotransferase) concentrations, and Child-Pugh scores were natural log-transformed because their distributions were skewed, and the geometric means (GMs) were compared. In this case, p-values were calculated after logarithmic transformation. Mean values of continuous variables in patients and controls were compared using Student's t-tests. Mean values of continuous variables for the four groups (controls 
Table 1. Demographic, clinical and laboratory features of the studied male subjects

\begin{tabular}{ccc}
\hline \hline & Liver cirrhotics $(\mathrm{n}=49)$ & Controls $(\mathrm{n}=23)$ \\
\hline Age (years) & $47.9 \pm 6.4^{*}$ & $52.7 \pm 5.2$ \\
\hline High signal intensity on MRI & $30(61.2 \%)^{*}$ & $2(8.7 \%)$ \\
\hline Pallidal index & $118.1 \pm 14.8^{*}$ & $105.8 \pm 3.8$ \\
\hline Blood manganese $(\mu \mathrm{g} / \mathrm{d} l)^{+}$ & $1.88(0.78-7.50)^{*}$ & $1.45(0.86-2.94)$ \\
\hline Hemoglobin $(\mathrm{g} / \mathrm{d} l)$ & $12.6 \pm 2.5^{*}$ & $14.8 \pm 1.1$ \\
\hline AST $(\mathrm{IU} / \mathrm{L})^{+}$ & $49.6(5-260)^{*}$ & $22.5(12-32)$ \\
\hline ALT $(\mathrm{IU} / \mathrm{L})^{+}$ & $50.1(15-581)^{*}$ & $24.6(10-51)$ \\
\hline Child-Pugh score & $7.5(5-14)$ & \\
\hline
\end{tabular}

NOTE, Data are mean \pm standard deviation or $^{+}$geometric mean (range). In the case of geometric mean, p-values were calculated by t-test after logarithmic transformation. ${ }^{*} \mathrm{p}<0.05$ vs control

AST; aspartate aminotransferase, ALT; alanine aminotransferase

Table 2. Pallidal index (PI) and blood manganese (Mn) concentrations by Child-Pugh classification

\begin{tabular}{ccccc}
\hline \hline & \multirow{2}{*}{ Controls $(\mathrm{n}=23)$} & \multicolumn{3}{c}{ Child-Pugh classification } \\
\cline { 3 - 5 } & & $\mathrm{A}(\mathrm{n}=27)$ & $\mathrm{B}(\mathrm{n}=11)$ & $\mathrm{C}(\mathrm{n}=11)$ \\
\hline PI & $105.8 \pm 3.8$ & $109.9 \pm 10.5$ & $125.7 \pm 15.1^{*}$ & $130.9 \pm 10.2^{* \#}$ \\
\hline Blood $\mathrm{Mn}(\mu \mathrm{g} / \mathrm{d} l)$ & $1.45(0.86-2.94)$ & $1.73(0.95-7.50)$ & $2.44(0.97-5.10)^{*}$ & $1.77(0.78-4.21)$ \\
\hline Proportion with high pallidal signal & $2(8.7 \%)$ & $9(33.3 \%)$ & $10(90.9 \%)$ & $11(100.0 \%)$ \\
\hline
\end{tabular}

NOTE, Data are mean \pm standard deviation or geometric mean (range). In the case of geometric mean, $p$-values were calculated by ANOVA with Scheffe's multiple comparison test after logarithmic transformation. ${ }^{*} \mathrm{p}<0.05$ vs control; ${ }^{*}$ : $p<0.05$ vs ChildPugh class A

and patient subgroups by Child-Pugh classification) were compared using one way analysis of variance (ANOVA). If the ANOVA showed significance at $\mathrm{P}$ $<0.05$, Scheffe's multiple comparison test was performed to identify the subgroup that was significantly different from the other subgroups. Multiple regression analysis was performed to verify any association between variables such as Mn, Child-Pugh class and AST/ALT (explanatory variables) and PI value (response variable) while controlling for the influence of other confounding variables such as age, smoking, and alcohol consumption.

\section{Results}

The general and clinical characteristics of the subjects are shown in Table 1. The mean age of the 49 cirrhotic patients was 47.9 years, all were male, and all had hepatitis B-induced liver cirrhosis. The mean age of the 23 male control subjects was 52.7 years; none had been exposed to $\mathrm{Mn}$ or had any neurological disease, liver disease, or anemia. We found that the GM of Mn concentration $(1.88 \mu \mathrm{g} / \mathrm{dl}$ vs. $1.45 \mu \mathrm{g} / \mathrm{dl}, \mathrm{p}<0.05)$ and mean PI (118.1 vs. 105.8, p $<0.05)$ were significantly higher in patients with cirrhosis than in controls. Increased T1-weighted MRI signals in the GP were observed in 30 of the $49(61.2 \%)$ patients with cirrhosis, compared with 2 of 23 (8.7\%) controls. AST (49.6 IU/L vs. $22.5 \mathrm{IU} / \mathrm{L}, \mathrm{p}$ $<0.05)$ and ALT (50.1 IU/L vs. $24.6 \mathrm{IU} / \mathrm{L}, \mathrm{p}<$ 0.05 ) concentrations were significantly higher, and hemoglobin concentrations $(12.6 \pm 2.5 \mathrm{~g} / \mathrm{dl}$ vs. $14.8 \pm$ $1.1 \mathrm{~g} / \mathrm{dl}, \mathrm{p}<0.05)$ were significantly lower, in liver cirrhotics than in controls. The GM of Child-Pugh score was 7.5 in the cirrhotics.

Mean PI differed significantly among four groups of individuals, three with liver cirrhosis (Child-Pugh classes A, B, and C) and the control group (Table 2). Mean PIs were significantly higher in patients with

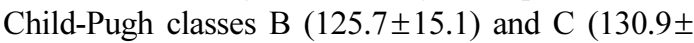
10.2) than in controls $(105.8 \pm 3.8)$ and was significantly higher in patients with Child-Pugh class C $(130.9 \pm 10.2)$ than in patients with Child-Pugh class A $(109.9 \pm 10.5)$. Mean blood Mn concentration 
Table 3. Multiple regression modeling of pallidal in$\operatorname{dex}(\mathrm{PI})$ in liver cirrhotics $(\mathrm{n}=47)$

\begin{tabular}{|c|c|c|}
\hline & $\begin{array}{c}\text { B Coefficient } \\
(95 \% \mathrm{CI})\end{array}$ & $\mathrm{R}^{2}$ \\
\hline \multicolumn{2}{|l|}{ Model 1} & \\
\hline Log blood manganese & $16.07(9.07-23.07)^{*}$ & \\
\hline \multicolumn{2}{|l|}{ Model 2} & \\
\hline Log blood manganese & $14.39(8.99-19.80)^{*}$ & $0.620^{*}$ \\
\hline Log Child-Pugh score & $22.55(14.39-30.70)^{*}$ & \\
\hline \multicolumn{2}{|l|}{ Model 3} & \\
\hline Log blood manganese & $15.10(9.78-20.41)^{*}$ & \\
\hline Child-Pugh class B vs A & $9.21(2.01-16.41)^{*}$ & $0.653^{*}$ \\
\hline Child-Pugh class C vs A & $20.59(13.92-27.26)^{*}$ & \\
\hline Child-Pugh class $\mathrm{C}$ vs $\mathrm{B}$ & $11.38(3.53-19.22)^{*}$ & \\
\hline \multicolumn{2}{|l|}{ Model 4} & \\
\hline Log blood manganese & $14.94(9.58-20.30)^{*}$ & \\
\hline Child-Pugh class B vs A & $9.21(2.01-16.41)^{*}$ & \\
\hline Child-Pugh class $\mathrm{C}$ vs $\mathrm{A}$ & $22.99(14.61-31.38)^{*}$ & $0.653 *$ \\
\hline Child-Pugh class $\mathrm{C}$ vs $\mathrm{B}$ & $11.46(3.39-19.52)^{*}$ & \\
\hline $\log$ AST & $-0.96(-7.23-5.31)$ & \\
\hline Log ALT & $-2.61(-7.81-2.59)$ & \\
\hline
\end{tabular}

Adjusted for age, alcohol drinking, and smoking. *: $\mathrm{p}<0.05$. AST; aspartate aminotransferase, ALT; alanine aminotransferase

was significantly higher in patients with Child-Pugh class B than in controls $(2.44 \mu \mathrm{g} / \mathrm{dl}$ vs. $1.45 \mu \mathrm{g} / \mathrm{dl}$, $\mathrm{p}<0.05)$. Increased pallidal signals were observed in $33.3 \%, 90.9 \%$, and $100 \%$ of patients with ChildPugh classes A, B, and $\mathrm{C}$, respectively, compared with $8.7 \%$ of controls.

Multiple linear regression analysis showed that log blood Mn was significantly associated with PI in cirrhotics, after controlling for age, smoking, drinking status, and Child-Pugh class $(\mathrm{p}<0.05)$ (Table 3, models 1-4). Child-Pugh score was also significantly associated with PI after controlling for Mn concentration, age, smoking, and drinking status $(\mathrm{p}<0.05)$ (Table 3, model 2). Patients with ChildPugh class $C$ had higher PI than those with ChildPugh class B as well as Child-Pugh class A after covariate adjustment. Patients with Child-Pugh class $\mathrm{B}$ also had higher PI than those with Child-Pugh class A after covariate adjustment (Table 3, models 3, 4). However, AST and ALT were not associated with PI after controlling for age, smoking, and drinking status (Table 3, model 4).

\section{Discussion}

Characteristic increased signal intensities, mainly confined to the GP, have been observed on T1weighted MRI after exposure to Mn. Chronic liver failure is also associated with increased signal intensities in the GP on T1-weighted MRI. ${ }^{5-7)}$ Liver cirrhosis prevents Mn clearance via biliary excretion due to a portal systemic shunt, resulting in increased blood $\mathrm{Mn}$ and increased signal intensity in GP even in patients not exposed to $\mathrm{Mn}^{1,2)}$ Our findings, of increased $\mathrm{T} 1$ signal intensities in the GP in $61.2 \%$ of our patients with hepatitis B-induced liver cirrhosis ${ }^{12,19,20)}$ and of a significant relationship between PI and blood Mn concentration, ${ }^{5-7,21)}$ were compatible with previous results. Moreover, we found that the severity of liver disease, as assessed by Child-Pugh score, was associated with the prevalence of increased $\mathrm{T} 1$ signals in the GP. PI was significantly higher in patients with Child-Pugh classes B and C than in the control group, and was significantly higher in patients with ChildPugh class $\mathrm{C}$ than in those with Child-Pugh class B. According to multiple linear regression analysis as well, patients with Child-Pugh class $\mathrm{C}$ had higher PI than those with Child-Pugh class B as well as ChildPugh class A after covariate adjustment. Furthermore, patients with Child-Pugh class B also had higher PI than those with Child-Pugh class A after covariate adjustment. Consistent with earlier results, ${ }^{11,12,22)}$ we observed a significant relationship between PI and the severity of the liver disease, as determined using the Child-Pugh scoring system. ${ }^{16)}$ Importantly, however, PI and blood Mn concentration did not differ in patients with early stage cirrhosis (i.e. Child-Pugh class A) and controls. We also found that pallidal signals were significantly associated with Child-Pugh score, but not with serum AST or ALT concentration. These results confirm and extend previous findings showing a correlation between increased pallidal signals and the severity of liver disease. The Child-Pugh scoring system includes the presence of ascites and encephalopathy, the concentrations of serum albumin and bilirubin, and prothrombin time. ${ }^{16}$

High pallidal T1 signals have been reported in children with congenital abnormalities of portalsystemic shunt due to dysgenesis of the portal venous system, but who showed almost normal liver function. ${ }^{23,24)}$ High pallidal signals have also been observed in patients with long standing portal- 
systemic shunt alone, due to portal vein thrombosis, without liver cirrhosis. ${ }^{25)}$ Animal models of portalsystemic shunt without cirrhosis have higher concentrations of $\mathrm{Mn}$ in the basal ganglia than in animal models of cirrhosis. ${ }^{13)}$ Moreover, high pallidal $\mathrm{T} 1$ signals were more prominent in patients with idiopathic portal hypertension than in patients with liver cirrhosis. ${ }^{26}$ Taken together, these findings support that pallidal signals is mainly affected by portal systemic shunt in advanced liver cirrhosis.

Pallidal signals have been reported to be highly prevalent in patients with liver cirrhosis (52$\left.100 \%) .{ }^{9} 16,19,20\right)$ Wide variation in the prevalence of high pallidal signals may be due to differences in the severity of liver diseases of the study subjects. The lower prevalence of high pallidal high signals among our patients than previously reported ${ }^{19-21)}$ may have been due to our inclusion of subjects with less severe liver disease.

Our findings have implications regarding occupational health. First, individuals susceptible to high pallidal signal include patients with liver cirrhosis, but not patients with chronic liver disease without portal systemic shunt, such as those with fatty liver disease or chronic hepatitis. Second, PI may be a surrogate marker of exposure to $\mathrm{Mn}$ and thus advanced liver cirrhosis may be a human model for manganism because the prevalence of liver cirrhosis is high. Patients with advanced cirrhosis have been documented with a form of parkinsonism with clinical symptoms similar to those of Mn-induced parkinsonism. 22,27-29) Our findings, however, are limited by our lack of information on subclinical neurobehavioral deficits in these individuals, because we did not perform neurobehavioral tests. In summary, we found that increased pallidal signals on T1-weighted MRI are mainly affected by the severity of liver disease.

\section{Acknowledgments}

This study was funded by Ulsan University Hospital (Biomedical Research Center Promotion Fund; UUH-07-14).

\section{References}

1. Kim Y. Neuroimaging in manganism. Neurotoxicology 2006; 27(3): 369-372.
2. Kim Y, Kim KS, Yang JS, Park IJ, Kim E, Jin Y, et al. Increase in signal intensities on T1-weighted magnetic resonance images in asymptomatic manganese-exposed workers. Neurotoxicology 1999; 20(6): 901-908.

3. Newland MC, Ceckler TL, Kordower JH, Weiss B. Visualizing manganese in the primate basal ganglia with magnetic resonance imaging. Exp Neurol 1989; 106(3): 251-258.

4. Nelson K, Golnick J, Korn T, Angle C. Manganese encephalopathy: utility of early magnetic resonance imaging. Br J Ind Med 1993; 50(6): 510513.

5. Butterworth RF, Spahr L, Fontaine S, Layrargues GP. Manganese toxicity, dopaminergic dysfunction and hepatic encephalopathy. Metab Brain Dis 1995; 10(4): 259-267.

6. Hauser RA, Zesiewicz TA, Martinez C, Rosemurgy AS, Olanow CW. Blood manganese correlates with brain magnetic resonance imaging changes in patients with liver disease. Can J Neurol Sci 1996; 23(2): 95-98.

7. Saito H, Ejima A. Liver dysfunction and probable manganese accumulation in the brainstem and basal ganglia. J Neurol Neurosurg Psychiatry 1995; 58(6): 760-761.

8. Krieger D, Krieger S, Jansen O, Gass P, Theilmann L, Lichtnecker H. Manganese and chronic hepatic encephalopathy. Lancet 1995; 346(8970): 270-274.

9. Maeda H, Sato M, Yoshikawa A, Kimura M, Sonomura T, Terada M, et al. Brain MR imaging in patients with hepatic cirrhosis: relationship between high intensity signal in basal ganglia on T1-weighted images and elemental concentrations in brain. Neuroradiology 1997; 39(8): 546-550.

10. Inoue E, Shinich H, Narumi Y, Fujita M, Kuriyama K, Kadota T, et al. Portal-systemic encephalopathy: presence of basal ganglia lesions with high signal intensity on MR images. Radiology 1991; 179(2): 551-555.

11. Kulisevsky J, Pujol J, Balanzo J, Junque C, Deus J, Capdevilla A, et al. Pallidal hyperintensity on magnetic resonance imaging in cirrhotic patients: clinical correlations. Hepatology 1992; 16(6): 13821388.

12. Pujol A, Pujol J, Graus F, Rimola A, Peri J, Mercader JM, et al. Hyperintense globus pallidus on T1-weighted MRI in cirrhotic patients is associated with severity of liver failure. Neurology 1993; 43(1): 65-69.

13. Rose C, Butterworth RF, Zayed J, Normandin L, Todd K, Michalak A, et al. Manganese deposition 
in basal ganglia structures results from both portalsystemic shunting and liver dysfunction. Gastroenterology 1999; 117(3): 640-644.

14. Thuluvath PJ, Edwin D, Yue NC, deVilliers C, Hochman S, Klein A. Increased signals seen in globus pallidus in T1-weighted magnetic resonance imaging in cirrhotics are not suggestive of chronic hepatic encephalopathy. Hepatology 1995; 21(2): 440-442.

15. Weissenborn K, Ehrenheim C, Hori A, Kubicka S, Manns MP. Pallidal lesions in patients with liver cirrhosis: clinical and MRI evaluation. Metab Brain Dis 1995; 10(3): 219-231.

16. Pugh RNH, Murray-Lyon IM, Dawson JL, Pietroni $\mathrm{MC}$, Williams R. Transection of the esophagus for bleeding esophageal varices. Br J Surg 1973; 60(8): 646-649.

17. Baldwin S, Deaker M, Maher W. Low volume microwave digestion of marine biological tissues for the measurement of trace elements. Analyst 1994; 119(8): 1701-1704.

18. Francois B, Oliver G, Josiane A, Francis P. Determination of manganese in biological materials by electrothermal atomic absorption spectrometry, a review. Clinical Chem 1988; 34(2): 227-234.

19. Choi Y, Park JK, Park NH, Shin JW, Yoo C-I, Lee $\mathrm{CR}$, et al. Whole blood and red blood cell manganese reflected signal intensities of T1-weighted MRI better than plasma manganese in liver cirrhotics. J Occup Health 2005; 47(1): 68-73.

20. Bang S-J, Choi SH, Park NH, Shin JW, Choy N, $\mathrm{Kim} \mathrm{SH}$, et al. High pallidal T1 signal is rarely observed in obstructive jaundice, but is frequently observed in liver cirrhosis. J Occup Health 2007; 49(4): 268-272.

21. Park NH, Park JK, Choi Y, Yoo C-I, Lee CR, Lee $\mathrm{H}$, et al. Whole blood manganese correlates with high signal intensities on T1-weighted MRI in patients with liver cirrhosis. Neurotoxicology 2003; 24(6): 909-915.

22. Kim J, Kim J-M, Kim YK, Shin JW, Choi SH, $\mathrm{Kim} \mathrm{SE}$, et al. Dopamine transporter SPECT of a liver cirrhotic with atypical parkinsonism. Ind Health 2007; 45(3): 497-500.

23. Yanai S, Minami T, Sonoda K, Gondo K, Tasaki $\mathrm{K}$, Hijii T, et al.: Patent ductus venosus associated with a hyperintense globus pallidum on T1weighted magnetic resonance imaging and pulmonary hypertension. Eur J Pediatr 1995; 154(7): 526-529.

24. Ihara K, Hijii T, Kuromaru R, Ariyoshi M, Kira R, Fukushige J, et al. High-intensity basal ganglia lesions on T1-weighted images in two toddlers with elevated blood manganese with portosystemic shunts. Neuroradiology 1999; 41(3): 195198.

25. Nolte W, Wiltfang J, Schindler CG, Unterberg K, Finkenstaedt M, Niedmann PD, et al. Bright basal ganglia in T1-weighted magnetic resonance images are frequent in patients with portal vein thrombosis without liver cirrhosis and not suggestive of hepatic encephalopathy. J Hepatol 1998; 29(3): 443-449.

26. Fukuzawa T, Matsutani S, Maruyama H, Akiike T, Saisho H, Hattori T. Magnetic resonance images of the globus pallidus in patients with idiopathic portal hypertension: a quantitative analysis of the relationship between signal intensity and the grade of portosystemic shunt. J Gastroenterol Hepatol 2006; 21(5): 902-907.

27. Kim JM, Kim JS, Jeong SH, Kim YK, Kim SE, $\mathrm{Kim} \mathrm{SH}$, et al. Dopaminergic neuronal integrity in parkinsonism associated with liver cirrhosis. Neurotoxicology 2010; 31(4): 351-355.

28. Klos KJ, Ahlskog JE, Kumar N, Cambern S, Butz $\mathrm{J}$, Burritt $\mathrm{M}$, et al. Brain metal concentrations in chronic liver failure patients and pallidal T1 MRI hyperintensity. Neurology 2006; 67(11): 1984-1989.

29. Racette BA, Antenor JA, McGee-Minninch L, Moerlein SM, Videen TO, Kotagal V, et al. [18F]FDOPA PET and clinical features in parkinsonism due to manganism. Mov Disord 2005; 20(4): 492-496. 